\title{
A Globally Convergent Algorithm of Variational Inequality
}

\author{
Chengjiang Yin \\ Linyi University at Feixian \\ Feixian, Shandong, P.R.China
}

\begin{abstract}
The algorithm of variational inequality is the important and valuable question in real life all the time. In this paper, a globally convergent algorithm of variational inequality is proposed. The method ensures that the corrector step sizes have a uniformly positive bound from below. In order to prove convergence of algorithm, we first establish some definitions, properties and theorem, and then we prove its global convergence under appropriate conditions.
\end{abstract}

Keywords-Iinfinite The variational inequality; Uniformly positive bound from below; Global convergence.

\section{INTRODUCTION}

Consider the generalized variational inequalities: we seek $u^{*} \in R^{n}$,that satisfies $g\left(u^{*}\right) \in \Omega$ and

(1)

$$
\left(g(u)-g\left(u^{*}\right)\right)^{T} F\left(u^{*}\right)>0, \forall g(u) \in \Omega
$$

Here $F: R^{n} \rightarrow R^{n}, G: R^{n} \rightarrow \Omega$ are given nonlinear operators and $\Omega \subseteq R^{n}$ is non-empty closed convex set. Noor had introduced problem (1)in his literature[1], and generalized variational inequalities is called GVI. GVI have important applications in economics and nonlinear analysis etc.

When $g(u) \equiv u$, GVI degenerate into VI: seek a vector $u^{*} \in \Omega$, that Satisfies $\left(u-u^{*}\right)^{T} F\left(u^{*}\right) \geq 0$ and $\forall u \in \Omega$.

Sun studied VI in his literature [2], and gave a new method to find the descent direction in the following way:

$$
d(u, \beta)=e(u, \beta)-\beta F(u)+\beta F\left(p_{\Omega}[u-\beta F(u)]\right)
$$

Where $e(u, \beta)=u-p_{\Omega}[u-\beta F(u)]$ and $p_{\Omega}$ is a rectangular projection from $R^{n}$ to $\Omega$.

Assuming that the underlying mapping $F$ is pseudomonotone, the author proves that this new method is globally convergent and gives a necessary and sufficient condition when the solution set is nonempty. Using literature thoughts [2-6], we propose a modified projection-type method of GVI.

\section{DEFINITION AND PROPERTIES}

In order to prove convergence of algorithm, we need someconclusions and assumptions, review that as fellows:
For a nonempty closed convex-set $\Omega \subseteq R^{n}$, where

$u \in R^{n}$, set $p_{\Omega}(u)$ is a rectangular projection from $u$ to $\Omega$, that means $p_{\Omega}(u)=\arg \min \{\mathrm{P} v-u \mathrm{P}, u, v \in \Omega\}$.

Some important properties of projection are as follows:

Lemma1 If $\Omega \subseteq R^{n}$ is a nonempty-closed convex set,for all $u \in R^{n}$ and $v \in \Omega$.then we have that

$$
\left\langle p_{\Omega}(u)-u, v-p_{\Omega}(u)\right\rangle \geq 0
$$

According to Lemmal we have a conclusion that projection operator is non-expansive,and we also obtain an equivalent proposition on solution set of GVI.

Lemma2 Vector $u^{*} \in R^{n}$ is a solution of GVI if and only if $g\left(u^{*}\right)=p_{\Omega}\left[g\left(u^{*}\right)-\beta F\left(u^{*}\right)\right]$, for all $\beta>0$.

Assumptions:

1) $\Omega^{*}$ is non-empty set : and $\Omega^{*} \in R^{n}$ is a solution set of GVI.

2) $F: R^{n} \rightarrow R^{n}$ is g- monotonous projection mapping, such that: $\langle F(u)-F(v), g(u)-g(v)\rangle \geq 0$, for all $u, v \in R^{n}$.

3) $g: R^{n} \rightarrow R^{n}$ is nonsingular mapping, there is a positive constant $\varphi$ satisfies $\mathrm{P} g(u)-g(v) \mathrm{P} \geq \varphi \mathrm{P} v-u$ P.for all $u, v \in R^{n}$

In this paper, we define residual vector $e(u, \beta)$ as fellows:

$$
e(u, \beta):=g(u)-p_{\Omega}[g(u)-\beta F(u)] .
$$

Lemma3 Suppose that $\Omega \subseteq R^{n}$ is a nonempty-closed convex set, we choose optional $u \in R^{n}$ and $\beta_{1}>\beta_{2}>0$, then we have
1) $\mathrm{P} e\left(u, \beta_{1}\right) \mathrm{P} \geq \mathrm{P} e\left(u, \beta_{2}\right) \mathrm{P}$;
2) $\mathrm{P} e\left(u, \beta_{1}\right) \mathrm{P} / \beta_{1} \geq \mathrm{P} e\left(u, \beta_{2}\right) \mathrm{P} / \beta_{2}$

\section{ALGORITHM AND CONVERGENCE}

\section{A. Algorithm}

Initial step:

set $u^{0} \in R^{n}$ and $g\left(u^{0}\right) \in \Omega$ and $\delta, \mu \in(0,1), \gamma \in(0,2)$. If $e\left(u^{0}, 1\right)=0$, then stop it; set $\mathrm{k}:=0$,otherwise. 
Iterative step:Set $\beta_{k}=\mu^{m_{k}}$ and $m_{k}$ is a minimal nonnegative integer $\mathrm{m}$ and satisfies formula below

$$
\mu^{m} \mathrm{P} F\left(g\left(u^{k}\right)\right)-F\left(g\left(u^{k}\right)-e\left(u^{k}, \mu^{m}\right)\right) \mathrm{P} \leq \delta \mathrm{P} e\left(u^{k}, \mu^{m}\right) \mathrm{P}
$$

Set $g\left(u^{k+1}\right)=p_{\Omega}\left[g\left(u^{k}\right)-\gamma \rho\left(u^{k}, \beta_{k}\right) d\left(u^{k}, \beta_{k}\right)\right]$,

where

$$
\begin{array}{r}
d\left(u^{k}, \beta_{k}\right)=e\left(u^{k}, \beta_{k}\right)-\beta_{k} F\left(g\left(u^{k}\right)\right)+\beta_{k} F\left(p_{\Omega}\left[g\left(u^{k}\right)-\beta_{k} F\left(u^{k}\right)\right]\right), \\
\rho\left(u^{k}, \beta_{k}\right)=(1-\delta) \mathrm{P} e\left(u^{k}, \beta_{k}\right) \mathrm{P}^{2} / \mathrm{P} d\left(u^{k}, \beta_{k}\right) \mathrm{P}^{2} .
\end{array}
$$

If $e\left(u^{k}, \beta_{k}\right)=0$, then stop; the iterative process continues otherwise.

Lemma4 Suppose $u \in R^{n}, g(u) \in \Omega$, and $u$ isn `t a solution of GVI, choose arbitrary $\delta \in(0,1)$,exist $\beta(u) \in(0,1]$,for all $\beta \in(0, \not{\beta}(u)]$,we have

$$
\beta \mathrm{P} F(g(u))-F(g(u)-e(u, \beta)) \mathrm{P} \leq \delta \mathrm{P} e(u, \beta) \mathrm{P}
$$

PROOF. Suppose that there is $\delta \in(0,1)$, for arbitrary $\beta \in(0,1]$, exist $\beta \in(0, \beta]$, it satisfies such that

$$
\beta \mathrm{P} F(g(u))-F(g(u)-e(u, \beta)) \mathrm{P}>\delta \mathrm{P} e(u, \beta) \mathrm{P}
$$

If $\beta_{1}=1$, there is $\beta_{1} \in\left(0, \beta_{1}\right]$, we obtain that

$$
\beta_{1} \mathrm{P} F(g(u))-F\left(g(u)-e\left(u, \beta_{1}\right)\right) \mathrm{P}>\delta \mathrm{P} e\left(u, \beta_{1}\right) \mathrm{P} .
$$

If $\vec{B}_{2}=\min \left\{1 / 2, \beta_{1}\right\}$, there is $\beta_{2} \in\left(0, \vec{B}_{2}\right]$, we obtain that

$$
\beta_{2} \mathrm{P} F(g(u))-F\left(g(u)-e\left(u, \beta_{2}\right)\right) \mathrm{P}>\delta \mathrm{P} e\left(u, \beta_{2}\right) \mathrm{P} .
$$

If $\vec{p}_{n}=\min \left\{1 / n, \beta_{n-1}\right\}$, there is $\beta_{n} \in\left(0, \vec{p}_{n}\right]$, we have that

$$
\beta_{n} \mathrm{P} F(g(u))-F\left(g(u)-e\left(u, \beta_{n}\right)\right) \mathrm{P}>\delta \mathrm{P} e\left(u, \beta_{n}\right) \mathrm{P}
$$

when $n \geq 1$, we obtain a sequence $\left\{\beta_{n}\right\}$, such that $\beta_{n} \leq \frac{1}{n}$ and

$$
\beta_{n} \mathrm{P} F(g(u))-F\left(g(u)-e\left(u, \beta_{n}\right)\right) \mathrm{P}>\delta \mathrm{P} e\left(u, \beta_{n}\right) \mathrm{P}
$$

From $g(u) \in \Omega$ and the expression of $e(u, \beta)$ and $\beta_{n} \leq 1 / n$, we have $\lim _{n \rightarrow \infty} e\left(u, \beta_{n}\right)=0$.According to formula(5)and the continuity of $F$ and the conclusion of Lemma3,we obtain that

$$
\begin{aligned}
& 0=\lim _{n \rightarrow \infty} \mathrm{P} F(g(u))-F\left(g(u)-e\left(u, \beta_{n}\right)\right) \mathrm{P} \geq \\
& \delta \lim _{n \rightarrow \infty}\left(\mathrm{P} e\left(u, \beta_{n}\right) \mathrm{P} / \beta_{n}\right) \geq \delta \mathrm{P} e(u, 1) \mathrm{P}
\end{aligned}
$$

By combining Lemma2, obviously, we have $u \in \Omega^{*}$, this is contradiction for the known .and the result is proved.

Lemma5 Suppose that $F(x)$ is g- monotonic mapping, we choose $u^{*} \in \Omega^{*}$ arbitrarily, when $u \in R^{n}$ and $g(u) \in \Omega$, we have that

$$
\left(g(u)-g\left(u^{*}\right)\right)^{T} d(u, \beta) \geq \mathrm{P} e(u, \beta) \mathrm{P}^{2}-
$$

$\beta e(u, \beta)^{T}(F(g(u)-F(g(u)-e(u, \beta)))$

PROOF. From (2),we obtain that

$$
\begin{gathered}
\left\{g(u)-\beta F(u)-p_{\Omega}[g(u)-\beta F(u)]\right\}^{T}\left\{p_{\Omega}[g(u)-\beta F(u)]-g\left(u^{*}\right)\right\} \geq 0 \\
\text { i.e. }(e(u, \beta)-\beta F(u))^{T}\left(g(u)-g\left(u^{*}\right)-e(u, \beta)\right) \geq 0,
\end{gathered}
$$

so we have that

$$
\begin{gathered}
e(u, \beta)^{T}\left(g(u)-g\left(u^{*}\right)\right) \geq \mathrm{P} e(u, \beta) \mathrm{P}^{2}+ \\
\beta F(u)^{T}\left(p_{\Omega}[g(u)-\beta F(u)]-g\left(u^{*}\right)\right)
\end{gathered}
$$

From g- monotonic properties of $\mathrm{F}$, we have

$\beta\left(F\left(p_{\Omega}[g(u)-\beta F(u)]\right)-F\left(g\left(u^{*}\right)\right)\right)^{T}\left(p_{\Omega}[g(u)-\beta F(u)]-g\left(u^{*}\right)\right) \geq 0$

From $u^{*} \in \Omega^{*}$ and $p_{\Omega}[g(u)-\beta F(u)] \in \Omega$, we have that

$$
\beta\left(p_{\Omega}[g(u)-\beta F(u)]-g\left(u^{*}\right)\right)^{T} F\left(g\left(u^{*}\right)\right) \geq 0
$$

$\operatorname{Add}(6),(7),(8)$, we obtain that

$$
\begin{gathered}
\{e(u, \beta)+\beta F(g(u)-e(u, \beta))\}^{T}\left(g(u)-g\left(u^{*}\right)\right) \geq \\
\mathrm{P} e(u, \beta) \mathrm{P}^{2}+\beta F(u)^{T}\left(g(u)-g\left(u^{*}\right)-e(u, \beta)\right)+ \\
\beta e(u, \beta)^{T} F(g(u)-e(u, \beta)) .
\end{gathered}
$$

By transposing and sorting, the proposition is founded.

According to algorithm design and Lemma5,For all $u^{k}$ and $u^{*} \in \Omega$, we have

$$
\left(g\left(u^{k}\right)-g\left(u^{*}\right)\right)^{T} d\left(u^{k}, \beta_{k}\right) \geq(1-\delta) \mathrm{P} e\left(u^{k}, \beta_{k}\right) \mathrm{P}^{2}
$$

The result is proved.

Theorem1 Suppose that $F(x)$ is a continuous and gmonotonic mapping projection, so the sequence $\left\{u^{k}\right\}$ generate by algorithm is bounded.

PROOF. Suppose $u^{*} \in \Omega^{*}$, then we have

$$
\begin{gathered}
\mathrm{P} g\left(u^{k+1}\right)-g\left(u^{*}\right) \mathrm{P}^{2} \leq \mathrm{P} g\left(u^{k}\right)-g\left(u^{*}\right)- \\
\gamma \rho\left(u^{k}, \beta_{k}\right) d\left(u^{k}, \beta_{k}\right) \mathrm{P}^{2}-\mathrm{P} g\left(u^{k}\right)-g\left(u^{*}\right) \mathrm{P}^{2} \\
-2 \gamma \rho\left(u^{k}, \beta_{k}\right)\left(g\left(u^{k}\right)-g\left(u^{*}\right)\right)^{T} d\left(u^{k}, \beta_{k}\right)+ \\
\gamma^{2} \rho\left(u^{k}, \beta_{k}\right)^{2} \mathrm{P} d\left(u^{k}, \beta_{k}\right) \mathrm{P}^{2} \leq
\end{gathered}
$$

$\mathrm{P} g\left(u^{k}\right)-g\left(u^{*}\right) \mathrm{P}^{2}-\gamma(2-\gamma)(1-\delta) \rho\left(u^{k}, \beta_{k}\right) \mathrm{P} e\left(u^{k}, \beta_{k}\right) \mathrm{P}^{2}$

For $\gamma \in(0,2)$ and $\delta \in(0,1)$, use the formula of above,we have that

$\mathrm{P} g\left(u^{k+1}\right)-g\left(u^{*}\right) \mathrm{P} \leq \mathrm{P} g\left(u^{k}\right)-g\left(u^{*}\right) \mathrm{P} \leq \mathrm{L} \leq \mathrm{P} g\left(u^{0}\right)-g\left(u^{*}\right) \mathrm{P}$

By combining assumption3), the sequence $\left\{u^{k}\right\}$ which generated by algorithm is bounded.The result is proved.

From theorem1,we obtain this algorithm is a projectiontype and contraction method.

Lemma6 $\rho\left(u^{k}, \beta_{k}\right)$ which is generated by algorithm is a uniformly positive bound from below. i.e. there is $\tau>0$, such that $\rho\left(u^{k}, \beta_{k}\right) \geq \tau$.

PROOF. From the expression of $d\left(u^{k}, \beta_{k}\right)$ and(3),we obtain that 


$$
\begin{gathered}
\mathrm{P} d\left(u^{k}, \beta_{k}\right) \mathrm{P}^{2} \leq 2 \mathrm{P} e\left(u^{k}, \beta_{k}\right) \mathrm{P}^{2}+2 \beta_{k}{ }^{2} \mathrm{P} F\left(g\left(u^{k}\right)\right)- \\
F\left(p_{\Omega}\left[g\left(u^{k}\right)-e\left(u^{k}, \beta_{k}\right)\right]\right) \mathrm{P}^{2} \leq 2\left(1+\delta^{2}\right) \mathrm{P} e\left(u^{k}, \beta_{k}\right) \mathrm{P}^{2}
\end{gathered}
$$

According to the expression of $\rho\left(u^{k}, \beta_{k}\right)$, we have

$$
\rho\left(u^{k}, \beta_{k}\right) \geq \frac{1-\delta}{2\left(1+\delta^{2}\right)}:=\tau
$$

So the step length of this algorithm is a uniformly positive bound from below. the result is proved.

From Lemma6 and Theorem1,we have that

$$
\begin{array}{r}
\mathrm{P} g\left(u^{k+1}\right)-g\left(u^{*}\right) \mathrm{P}^{2} \leq \mathrm{P} g\left(u^{k}\right)-g\left(u^{*}\right) \mathrm{P}^{2}- \\
\tau \gamma(2-\gamma)(1-\delta) \mathrm{P} e\left(u^{k}, \beta_{k}\right) \mathrm{P}^{2}
\end{array}
$$

Such that

$$
\sum_{k=0}^{\infty} \mathrm{P} e\left(u^{k}, \beta_{k}\right) \mathrm{P}^{2} \leq \infty \text { i.e. } \lim _{k \rightarrow \infty} \mathrm{P} e\left(u^{k}, \beta_{k}\right) \mathrm{P}=0
$$

2.2 Proof of the global convergence

Theorem2 Suppose that the conditions of theorem1 holds, we have that

1) $\left.\lim _{k \rightarrow \infty} \mathrm{P} e\left(u^{k}, \beta_{k}\right) \mathrm{P} / \beta_{k}=0 ; 2\right) \quad\left\{u^{k}\right\}$ converges to the solution of GVI.

PROOF. 1) Suppose that there is a boundless index subset $K_{0}$,we have

$$
\mathrm{P} e\left(u^{k}, \beta_{k}\right) \mathrm{P} / \beta_{k} \geq \varepsilon>0 \quad \text { for all } k \in K_{0},
$$

by combining $\lim _{k \rightarrow \infty} \mathrm{P} e\left(u^{k}, \beta_{k}\right) \mathrm{P}=0$, we obtain that

$\lim _{x \rightarrow \infty, k \in K_{0}} \beta_{k}=0$, because $\left\{u^{k}\right\}$ is bounded, so $\left\{F\left(u^{k}\right)\right\}$ is bounded too, according to non-expansivity of projection algorithm, we obtain that

$$
\begin{aligned}
& \lim _{x \rightarrow \infty, k \in K_{0}} \mathrm{P} g\left(u^{k}\right)-p_{\Omega}\left[g\left(u^{k}\right)-\beta_{k} F\left(u^{k}\right) / \mu\right] \mathrm{P} \leq \\
& \lim _{x \rightarrow \infty, k \in K_{0}} \beta_{k} \mathrm{P} F\left(u^{k}\right) \mathrm{P} / \mu=0,
\end{aligned}
$$

by combining the continuity of $F$ and the conclusion of lemma3 and liner search (3),for all $k \in K_{0}$ and $k \rightarrow \infty$ we obtain that

$$
\begin{gathered}
0 \leftarrow \mathrm{P} F\left(g\left(u^{k}\right)\right)-F\left(p_{\Omega}\left[g\left(u^{k}\right)-\beta_{k} F\left(u^{k}\right) / \mu\right]\right) \mathrm{P}> \\
\delta \frac{\mathrm{P} e\left(u^{k}, \beta_{k} / \mu\right) \mathrm{P}}{\beta_{k} / \mu} \geq \mu \delta \frac{\mathrm{P} e\left(u^{k}, \beta_{k}\right) \mathrm{P}}{\beta_{k}}
\end{gathered}
$$

this is contradiction for the assumptions ,then the result is proved.

2) Divide two kinds of case

i) $\limsup _{k \rightarrow \infty} \beta_{k}>0$ i.e. there is $\varepsilon_{0}>0$ and $K_{1}$, such that $\beta_{k} \geq \varepsilon_{0}$, $k \in K_{1}$.

From $\mathrm{P} e\left(u^{k}, \beta_{k}\right) \mathrm{P} \geq \mathrm{P} e\left(u^{k}, \varepsilon_{0}\right) \mathrm{P}$ and $k \in K_{1}$ and (10) we have that $\mathrm{P} e\left(u^{k}, \varepsilon_{0}\right) \mathrm{P} \rightarrow 0$.

Because $\left\{u^{k}\right\}$ is bounded, so we ensure that there is a convergent subset $\left\{u^{k_{j}}\right\}$, and suppose its limiting point is $\bar{u}$.By combining the continuity of $\mathrm{P} e\left(u, \varepsilon_{0}\right) \mathrm{P}$,we obtain that

$$
\mathrm{P} e\left(\bar{u}, \varepsilon_{0}\right) \mathrm{P}=0 \text {, means } \bar{u} \in \Omega^{*} \text {. }
$$

Proof of global convergence is below. From theorem1,we have

$$
\mathrm{P} g\left(u^{k+1}\right)-g(\bar{u}) \mathrm{P} \leq \mathrm{P} g\left(u^{k}\right)-g(\bar{u}) \mathrm{P},
$$

we choose $\hat{u}$ arbitrarily, $\hat{u}$ is an accumulation point of $\left\{u^{k}\right\}$, and $\left\{u^{k_{l}}\right\}$ is a convergence subsequence, there is $k_{l}>k_{j}$ for all $k_{j}$, such that

$\mathrm{P} g\left(u^{k_{l}}\right)-g(\bar{u}) \mathrm{P} \leq \mathrm{P} g\left(u^{k_{j}}\right)-g(\bar{u}) \mathrm{P}$, set $k_{j} \rightarrow \infty$,by combining $k_{l}>k_{j}$, we have

$\mathrm{P} g(\overleftrightarrow{\mathbb{u}})-g(\bar{u}) \mathrm{P} \leq \mathrm{P} g(\bar{u})-g(\bar{u}) \mathrm{P}=0$.

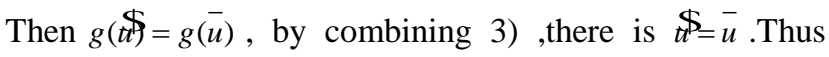
sequence $\left\{u^{k}\right\}$ global converge to $\bar{u}$.

ii) $\lim _{k \rightarrow \infty} \beta_{k}=0$,from the conclusion 2) of lemma3, when $\mathrm{k}$ is big enough, we obtain that

$$
\frac{\mathrm{P} e\left(u^{k}, \beta_{k}\right) \mathrm{P}}{\beta_{k}} \geq \mathrm{P} e\left(u^{k}, 1\right) \mathrm{P},
$$

from the conclusion 1) of theorem 2 and the formula of above, we have that $\mathrm{P} e\left(u^{k}, 1\right) \mathrm{P} \rightarrow 0$.

From the similarly analysis as i) we proved theorem.

\section{CONCLUSIONS AND PROSPECT}

As everyone knows, variational inequality originated in the mathematical physics problem with the nonlinear programming, In this paper, we get a new globally convergent algorithm of variational inequality, it can be widely used in the physical, mechanical, engineering, economic and other fields ,how to popularize It better in Practical Engineering Application is a topic for future research.

\section{ACKNOWLEDGMENT}

The authors wish to give their sincere thanks to the anonymous referees for their valuable suggestions and helpful comments which improved the presentation of the paper.

\section{REFERENCES}

[1] NOOR M.A. General Variational Inequalities[J]. Appl Math Lett,1988,1(2):119-121.

[2] SUN D. A Class of Iterative Methods for solving Nonlinear Projection Equations[J]. J Optim Theory Appl,1996,91:123-140

[3] XIU N H,ZHANG J Z. Global projective-type error Bound for generalized variational inequalities [J]. J Optim Theory and Appl,2002,112(1:213-228)

[4] SOLODOV M V. Convergence Rate Analysis of Iteractive Algorithms for solving Variational Inequality Problems[J].Math Programming,2003,96:513-528

[5] Cheng X D. In Banach space reflexive solutions of a class of variational inequalities are given[J].Chongqing normal college journals.1991,16(2):44-48

[6] Wang C W. A variational inequalities problem solving the projection algorithm[J].Chongqing normal college journals.2005,22(1):6-1 